\title{
Cranial Nerve Involvement and Dysautonomia in Post-COVID-19 Guillain-Barré Syndrome
}

\author{
Toshiyuki Kakumoto ${ }^{1}$, Satoshi Kobayashi ${ }^{1}$, Hayato Yuuki ${ }^{1}$, Mitsuhiro Kainaga ${ }^{1}$, \\ Yuichiro Shirota ${ }^{1,2}$, Masashi Hamada ${ }^{1}$, Meiko Hashimoto Maeda ${ }^{1}$, Akatsuki Kubota ${ }^{1}$, \\ Mizuho Kawai ${ }^{3}$, Masaaki Saito ${ }^{3}$, Hiroyuki Ishiura ${ }^{1}$ and Tatsushi Toda ${ }^{1}$
}

\begin{abstract}
:
The clinical characteristics of Guillain-Barré syndrome (GBS) after coronavirus disease 2019 (COVID-19) remain unclear due to the small number of cases. We herein report a case of a Japanese patient with postCOVID-19 GBS who presented with facial and limb muscle weakness, sensory deficits, and autonomic dysfunction. Nerve conduction studies revealed demyelination. Head magnetic resonance imaging showed contrast enhancement in the bilateral facial nerves. Systemic management, including intubation, intravenous immunoglobulin therapy, and rehabilitation, improved the patient's condition. This was the first Japanese case of acute inflammatory demyelinating polyneuropathy after COVID-19 and was characterized by autonomic dysfunction and facial nerve enhancement.
\end{abstract}

Key words: COVID-19, Guillain-Barré syndrome, dysautonomia

(Intern Med 60: 3477-3480, 2021)

(DOI: 10.2169/internalmedicine.7355-21)

\section{Introduction}

The novel coronavirus infection, coronavirus disease 2019 (COVID-19), first appeared in Wuhan, China, in December 2019 and has since spread worldwide, causing a pandemic. COVID-19 manifests with cough, a fever, and dyspnea, and may lead to severe pneumonia. Some patients with COVID19 also present with neurological complications. GuillainBarré syndrome (GBS) often develops after infection and has also been reported after COVID-19 (1).

While several reports have observed the relationship between COVID-19 and the occurrence of GBS, the number of cases is small, especially in East Asia (1), making it difficult to characterize the clinical spectra of COVID-19associated GBS. Furthermore, the clinical characteristics of GBS differ substantially among populations (2).

We herein report a Japanese case of post-COVID-19 GBS that presented with dysautonomia and cranial nerve involvement accompanied by contrast enhancement on the bilateral facial nerves by magnetic resonance imaging (MRI).

\section{Case Report}

A 22-year-old previously healthy man was admitted to our hospital complaining of progressive weakness of the limbs and difficulty in urination and defecation. Twenty-two days before admission, he had a fever of $38^{\circ} \mathrm{C}$. He was diagnosed with COVID-19 after testing positive for severe acute respiratory syndrome coronavirus 2 (SARS-CoV-2) by real-time reverse transcriptase-polymerase chain reaction (RT-PCR) using nasopharyngeal swab fluid and was admitted to another hospital. The route of infection was unknown. He was discharged 10 days later without any specific treatment. Sixteen days after the onset of COVID-19 (which we consider day 1), he had stopped defecating. On day 3, limb weakness and hypesthesia of the extremities appeared and progressed gradually. His inability to urinate developed on day 6.

On admission, he was unable to walk, with a medical re-

${ }^{1}$ Department of Neurology, Graduate School of Medicine, The University of Tokyo, Japan, ${ }^{2}$ Department of Clinical Laboratory, The University of Tokyo Hospital, Japan and ${ }^{3}$ Department of Neurology, Tokyo Kyosai Hospital, Japan

Received: February 16, 2021; Accepted: July 8, 2021; Advance Publication by J-STAGE: August 24, 2021

Correspondence to Dr. Toshiyuki Kakumoto, toshikakumoto@gmail.com 


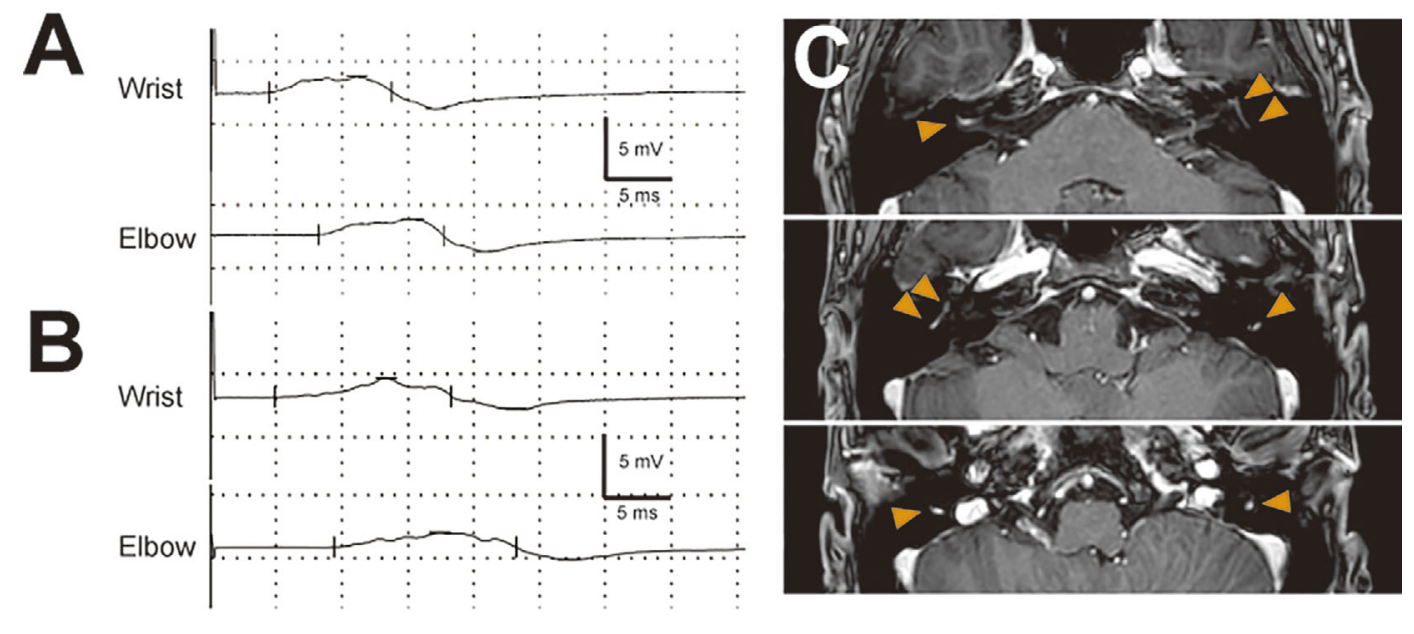

Figure 1. A, B: Right median motor nerve conduction study on days 8 (A) and 14 (B) after the onset of defecation. The upper and lower parts show wrist and elbow stimulation, respectively. The amplitudes of the compound muscle action potentials are decreased to $2.6 \mathrm{mV}(\mathrm{A})$ and $2.5 \mathrm{mV}(\mathrm{B})$, respectively, from peak to peak. Abnormal temporal dispersion was observed on day 8 (A) and was more prominent on day 14 than at other points (B). C: Contrast-enhanced T1-weighted magnetic resonance imaging of the head on day 25. Contrast enhancement is visible from the distal part of the meatal segment to the mastoid segment of the bilateral facial nerves (orange arrowheads).

Table 1. Nerve Conduction Study on Day 14 Following the Onset of Neurological Symptoms.

\begin{tabular}{lccccc}
\hline $\begin{array}{c}\text { Motor } \\
\text { Nerve }\end{array}$ & Side & $\begin{array}{c}\text { Distal latency } \\
(\mathrm{ms})\end{array}$ & $\begin{array}{c}\text { Amplitude } \\
(\mathrm{mV})\end{array}$ & $\begin{array}{c}\text { Velocity } \\
(\mathrm{m} / \mathrm{s})\end{array}$ & $\begin{array}{c}\text { F-wave frequency } \\
(\%)\end{array}$ \\
\hline Median & Right & 4.9 & $2.5 / 2.1$ & 48.3 & 68.8 \\
Ulnar & Right & 3.4 & $7.9 / 7.1$ & 61.2 & Not tested \\
Tibial & Left & 3.7 & $5.1 / 3.9$ & 42.9 & 0 \\
Tibial & Right & 5.3 & $3.4 / 3.1$ & 53.6 & 0 \\
Peroneal & Left & 8.2 & $2.3 / 1.4$ & 34.4 & Not tested \\
Peroneal & Right & 11.2 & $1.8 / 1.2$ & 41.3 & Not tested \\
\hline Sensory & Side & Latency & Amplitude & Velocity & \\
Nerve & & $(\mathrm{ms})$ & $(\mu \mathrm{V})$ & $(\mathrm{m} / \mathrm{s})$ & \\
\hline Median & Right & ND & ND & ND & \\
Ulnar & Right & 2.5 & 0.3 & 41.4 & \\
Sural & Left & 2.2 & 6.5 & 68.5 & \\
Sural & Right & 2.4 & 11.9 & 66.0 & \\
\hline
\end{tabular}

For motor studies, distal and proximal amplitudes are presented in this order; for each, stimuli were delivered at the wrist and elbow (upper limb) or at the ankle and knee (lower limb). ND: not detected

search council (MRC) grade of $<4$ in his lower limbs. Bilateral facial muscle weakness, dysarthria, and dysphagia were observed. The straight leg raising test was positive bilaterally. No tendon reflexes were observed. He also exhibited decreased superficial sensation, decreased vibratory sensation, and dysesthesia in the extremities and region corresponding to the ophthalmic division of the left trigeminal nerve. His pupils were bilaterally enlarged to $6 \mathrm{~mm}$ and responded promptly to light. The patient's blood pressure in the supine position was $157 / 102 \mathrm{mmHg}$ and increased to more than $200 \mathrm{mmHg}$ when the lower limbs were elevated. He exhibited sinus arrhythmia, with the heart rate fluctuating from 70 to $140 \mathrm{bpm}$. His Erasmus GBS respiratory in- sufficiency score (3) was four. Cerebrospinal fluid (CSF) obtained on day 7 showed marked albuminocytologic dissociation (white blood cell count, 5/mL; total protein, $307 \mathrm{mg}$ / $\mathrm{dL}$ ). The patient's immunoglobulin $\mathrm{G}$ ( $\mathrm{IgG}$ ) index was 0.70 . He was negative for serum $\operatorname{IgG}$ and $\operatorname{IgM}$ antibodies against GM1, GM2, GM3, GD1a, GD1b, GD3, GT1a, GT1b, GQ1 b, asialo-GM1, and galactocerebroside (Gal-C). Nerve conduction studies revealed demyelinating neuropathy (Fig. 1A, B, Table 1). The diagnosis of acute inflammatory demyelinating polyneuropathy (AIDP) was made based on Ho et al.'s criteria (4). No examinations for Campylobacter, Mycoplasma, Epstein-Barr virus, cytomegalovirus, or hepatitis $\mathrm{E}$ virus were performed. 


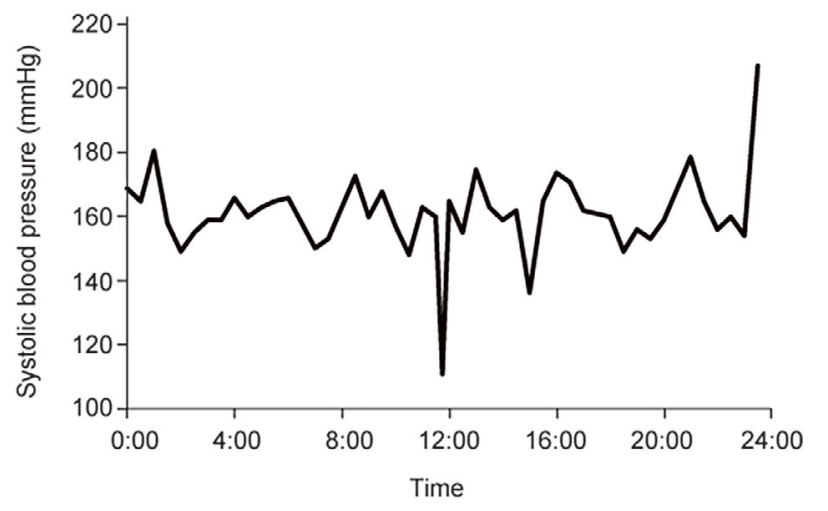

Figure 2. Systolic blood pressure monitoring by arterial line on day 8 . The patient's blood pressure was high and fluctuated spontaneously and when the body posture changed.

From the day of admission, intravenous immunoglobulin was administered (400 mg/kg/day for 5 days), and the patient was intubated and managed on a ventilator. One week later, he began to recover. He was extubated on day 18 . With physical therapy, he was able to walk with assistance at five weeks after the onset of neurological symptoms. He also recovered sensation, with only numbness in his hands remaining. Head MRI revealed contrast enhancement on the bilateral facial nerves (Fig. 1C), although lumbar MRI did not show any contrast enhancement on the nerve roots or leptomeninges. Regarding the autonomic system evaluation, topical $0.125 \%$ pilocarpine administration to the right eye caused miosis on day 22, suggesting parasympathetic postganglionic damage. The blood pressure measured at the radial arterial line was consistently high and fluctuated with changes in body posture (Fig. 2). The coefficient of variation of the R-R interval in the electrocardiogram fluctuated substantially during the treatment period, although it remained within the normal range $3.40 \%$ on admission, $6.93 \%$ on day $9,2.58 \%$ on day $15,4.05 \%$ on day $21,4.42 \%$ on day $27,6.46 \%$ on day 34 , and $6.42 \%$ on day 41$) .{ }^{123} \mathrm{I}-$ metaiodobenzylguanidine myocardial scintigraphy showed no abnormalities. Serum anti-ganglionic acetylcholine receptor antibody levels were not examined.

\section{Discussion}

We herein report a patient with post-COVID-19 GBS. The patient developed GBS 16 days after the onset of COVID19. He was negative for serum anti-glycolipid antibodies, a CSF examination showed albuminocytologic dissociation, and SARS-CoV-2 PCR in the CSF was negative. These findings are consistent with the features of reported cases of GBS after COVID-19 (1). The characteristics of this patient were autonomic dysfunction and contrast enhancement effect of the bilateral facial nerves on head MRI.

Dysautonomia is rarely observed in COVID-19-associated GBS (1). In a previous case series, among 73 cases, 4 exhibited bladder or bowel dysfunction, 4 exhibited tachycardia or unstable blood pressure, and 2 exhibited both (1).
Anti-ganglionic acetylcholine receptor antibody titers were not reported in these cases. Autonomic dysfunction can precede muscle weakness, as in our patient and as previously reported (5). The evaluation of the autonomic nerve function is limited partly because of the need to isolate patients with COVID-19. Our case was the first reported to have positive findings on a pilocarpine test, indicating parasympathetic nerve dysfunction in GBS after COVID-19. The patient also had marked fluctuations in blood pressure, as monitored by arterial lines, for 24 hours and changes in RR intervals over time (Fig. 2). His autonomic dysfunction gradually improved as his weakness and sensory deficits recovered, consistent with previous reports (5).

Another characteristic of this case was gadolinium enhancement of the bilateral facial nerves (Fig. 1C). In previous reports, 23 post-COVID-19 GBS patients underwent head MRI, with $4(17.4 \%)$ showing contrast-enhancing effects on the cranial nerves (1, 6-9). Autonomic dysfunction was not described in any of these cases. Among 10 nonCOVID-19-associated GBS patients treated at our hospital from 2005 to 2020 who underwent contrast-enhanced MRI, 3 had cranial nerve contrast enhancement (30\%), and 1 had autonomic dysfunction, such as tachycardia and constipation $(10 \%)$. As in the present patient, enhancement of the cranial nerves, including the facial nerves, on MRI was also observed in GBS in general.

The mechanism by which GBS develops after COVID-19 remains unclear. It could be a direct viral invasion of the nervous system or an abnormal immune-mediated response. In the present patient, RT-PCR for SARS-CoV-2 in the CSF sample was negative, whereas that of the nasal swab sample was still positive on admission. These results were similar to those of previous reports, and there was no evidence of direct invasion of the nervous system by SARS-CoV-2 (1). The high prevalence of albuminocytologic dissociation (71\%) suggests an abnormal immune response. Serum antiganglioside antibody positivity was low in GBS after COVID-19 (5.7\%) (1), which may suggest the presence of an unknown immune-mediated response. Three cases of post-COVID-19 GBS were reported to be positive for antiglycolipid antibodies, with anti-GM2 IgG/IgM, anti-GD1b IgG, and anti-Gal-C antibodies detected (10-12), whereas these antibodies were not detected in the present patient.

There have been no reports of demyelination findings in GBS after COVID-19 in Japan, except for the present patient (Table 2). Two Japanese patients with COVID-19associated GBS have been reported: one was classified as axonal type based on the results of nerve conduction studies, while the other was not classified, as a nerve conduction study could not be sufficiently carried out $(10,13)$. In Western Europe, 78\% (39/50) of GBS cases after COVID-19 showed demyelinating disease, which is comparable to the proportion of demyelinating forms of GBS unrelated to COVID-19 (69\%) $(1,14)$. The incidence of axonal-type GBS is known to have been higher in Japan than in Western countries before the COVID-19 era (2). However, whether 
Table 2. GBS Cases after COVID-19 Reported in Japan.

\begin{tabular}{|c|c|c|c|c|c|c|c|c|}
\hline Reference & $\begin{array}{l}\text { Age } \\
(\mathrm{y})\end{array}$ & Sex & Clinical features & $\begin{array}{l}\text { Autonomic } \\
\text { dysfunction }\end{array}$ & $\begin{array}{l}\text { Gd enhancement } \\
\text { on brain MRI }\end{array}$ & $\begin{array}{c}\text { GBS } \\
\text { electrophysiological } \\
\text { subtype }\end{array}$ & $\begin{array}{l}\text { Serum anti- } \\
\text { glycolipid } \\
\text { antibody }\end{array}$ & $\begin{array}{l}\text { Cerebrospinal } \\
\text { fluid }\end{array}$ \\
\hline (10) & 69 & M & $\begin{array}{l}\text { Absent cough reflex, } \\
\text { limb weakness, limb } \\
\text { areflexia }\end{array}$ & Unremarkable & Not tested & Not described & $\begin{array}{c}\text { anti-Gal-C } \\
\text { antibody }\end{array}$ & $\begin{array}{c}\text { TP } 202 \mathrm{mg} / \mathrm{dL} \\
\text { cell } 1 / \mu \mathrm{L}\end{array}$ \\
\hline (13) & 54 & $\mathrm{~F}$ & $\begin{array}{l}\text { Limb weakness, } \\
\text { numbness, lower } \\
\text { limb areflexia }\end{array}$ & $\begin{array}{c}\text { Not } \\
\text { documented }\end{array}$ & Not tested & Axonal type & Negative & $\begin{array}{l}\text { Normal protein } \\
\text { levels and cell } \\
\text { counts }\end{array}$ \\
\hline Present case & 22 & M & $\begin{array}{l}\text { Limb weakness, } \\
\text { limb hypoesthesia, } \\
\text { dysarthria, } \\
\text { dysphagia, left-sided } \\
\text { facial numbness, } \\
\text { dysautonomia }\end{array}$ & $\begin{array}{c}\text { Positive } \\
\text { pilocarpine } \\
\text { test, marked } \\
\text { fluctuations in } \\
\text { blood pressure } \\
\text { and heart rate }\end{array}$ & $\begin{array}{c}\text { Bilateral facial } \\
\text { nerves }\end{array}$ & AIDP & Negative & $\begin{array}{c}\mathrm{TP} 307 \mathrm{mg} / \mathrm{dL} \\
\text { cell } 5 / \mu \mathrm{L}\end{array}$ \\
\hline
\end{tabular}

GBS: Guillain-Barré syndrome, COVID-19: coronavirus disease 2019, Gd: gadolinium, Gal-C: galactocerebroside, TP: total protein, SARS-CoV-2: severe acute respiratory syndrome coronavirus 2, PCR: polymerase chain reaction, AIDP: acute inflammatory demyelinating polyneuropathy, M: male, F: female

or not genetic variations between populations affect the electrophysiological subtypes of COVID-19-associated GBS remains unclear. Since sensitizing antigens are considered to be shared by GBS after COVID-19, electrophysiological classification of COVID-19-associated GBS in Japan may provide insight into which factors - genetic variations among populations or infectious pathogens - mainly contribute to the subtype of GBS. In addition to the electrophysiological subtypes, the three cases of GBS after COVID-19 in Japan did not show common features in terms of age, clinical features, presence of anti-glycolipid antibodies, or CSF protein levels (Table 2). Such differing clinical features suggest that the mechanisms underlying GBS associated with SARSCoV-2 are various. The number of COVID-19-associated GBS cases in Japan is small, and the accumulation of additional cases is awaited.

The authors state that they have no Conflict of Interest (COI).

\section{References}

1. Abu-Rumeileh S, Abdelhak A, Foschi M, Tumani H, Otto M. Guillain-Barré syndrome spectrum associated with COVID-19: an up-to-date systematic review of 73 cases. J Neurol 2020.

2. Mitsui $Y$, Kusunoki S, Arimura K, et al. A multicentre prospective study of Guillain-Barré syndrome in Japan: a focus on the incidence of subtypes. J Neurol Neurosurg Psychiatry 86: 110-114, 2015.

3. Walgaard C, Lingsma HF, Ruts L, et al. Prediction of respiratory insufficiency in Guillain-Barré syndrome. Ann Neurol 67: 781787, 2010.

4. Ho TW, Mishu B, Li CY, et al. Guillain-Barré syndrome in northern China. Relationship to Campylobacter jejuni infection and anti-glycolipid antibodies. Brain 118: 597-605, 1995.

5. Ghosh R, Roy D, Sengupta S, et al. Autonomic dysfunction heralding acute motor axonal neuropathy in COVID-19. Int Clin Neurosci J 20: 1759-1761, 2020.

6. Bigaut K, Mallaret M, Baloglu S, et al. Guillain-Barré syndrome related to SARS-CoV-2 infection. Neurol Neuroimmunol Neuroinflamm 7: 2020

7. Chan JL, Ebadi H, Sarna JR. Guillain-Barré syndrome with facial diplegia related to SARS-CoV-2 infection. Can J Neurol Sci 47: 852-854, 2020.

8. Hutchins KL, Jansen JH, Comer AD, et al. COVID-19-associated bifacial weakness with paresthesia subtype of Guillain-Barré syndrome. Am J Neuroradiol 41: 1707-1711, 2020.

9. Toscano G, Palmerini F, Ravaglia S, et al. Guillain-Barré syndrome associated with SARS-CoV-2. N Engl J Med 382: 25742576, 2020.

10. Wada S, Nagasaki Y, Arimizu Y, et al. Neurological disorders identified during treatment of a SARS-CoV-2 infection. Intern Med 59: 2187-2189, 2020.

11. Gutiérrez-Ortiz C, Méndez-Guerrero A, Rodrigo-Rey S, et al. Miller Fisher syndrome and polyneuritis cranialis in COVID-19. Neurology 95: e601-e605, 2020.

12. Chan M, Han SC, Kelly S, Tamimi M, Giglio B, Lewis A. A case series of Guillain-Barré syndrome following COVID-19 infection in New York. Neurol Clin Pract 2020.

13. Hirayama T, Hongo Y, Kaida K, Kano O. Guillain-Barré syndrome after COVID-19 in Japan. BMJ Case Rep 13: 1-4, 2020.

14. Hadden RD, Cornblath DR, Hughes RA, et al.; Plasma Exchange/ Sandoglobulin Guillain-Barré Syndrome Trial Group. Electrophysiological classification of Guillain-Barré syndrome: clinical associations and outcome. Ann Neurol 44: 780-788, 1998.

The Internal Medicine is an Open Access journal distributed under the Creative Commons Attribution-NonCommercial-NoDerivatives 4.0 International License. To view the details of this license, please visit (https://creativecommons.org/licenses/ by-nc-nd/4.0/).

(C) 2021 The Japanese Society of Internal Medicine Intern Med 60: 3477-3480, 2021 